The population of Palestine amounts to about 300000 , Transjordan has about 400000 inhabitants and there are besides more than 500000 refugees living in the country.

Jordan has few natural riches. Main resources are agriculture (mediterranean products: wheat, grapes, figs, olives, tomatoes, etc.) and cattle raising (mostly sheep, goats and camels). Industry (oil, soap, textiles, etc.) is of little importance. Trade is not developped. No oil has ever been found. Jordan is therefore rather poor and it does not seem likely that it might ever be rich.

\title{
ZUR WASSERWIRTSCHAFT DES OUED RHIR IN DER ALGERISCHEN SAHARA
}

\author{
Karl Suter
}

Die Region des Oued Rhir mit 'Touggourt als wichtigstem Ort bildet das bedeutendste Dattelpalmengebiet der Sahara. Die bebaute Fläche ihrer 38 Oasen mißt gegenwärtig rund 10000 Hektaren.

Sie war, namentlich wegen ihrer besondern Grundwasserverhältnisse, schon wiederholt Gegenstand eingehender Untersuchungen, so von G. Rolland ${ }^{1}$, D. Moulias ${ }^{2}$, J. Savornin ${ }^{3}$. Auch widmet ihr R. CAPOT-REY ${ }^{4}$ in seinem Werk "Le Sahara Français" eine besondere Darstellung. Die folgende Ausführung, die sich auf einen persönlichen Augenschein an Ort und Stelle und auf eine Kontaktnahme mit dem Amt für Wasserwirtschaft in Touggourt stützt, ist als Ergänzung gedacht.

Das Tal des Oued Rhir beginnt im Süden bei den Palmengärten von Goug und Blidet Amor (85 m ü. M.), wo sich die Täler des Mya und des Igharghar miteinander vereinigen, und endet im Norden im Schott Melrhir $(-31 \mathrm{~m})$. Es ist $150 \mathrm{~km}$ lang. Sein Gefälle ist gering, beträgt es doch im ganzen bloß $116 \mathrm{~m}$, d.h. durchschnittlich $80 \mathrm{~cm}$ je Kilometer. Seine am tiefsten gelegene Oase ist Dendouga im Osten von Mraier auf dem Westrand des Schott Merouane mit - $26 \mathrm{~m}$. Seine geologisch-hydrologischen Verhältnisse sind noch nicht abgeklärt. Vielleicht bestand hier einst ein sich von Süden nach Norden wälzender Wüstenfluß, doch welcher? War es der Oued Mya, dessen weites Tal im östlichen Abschnitt der Hochfläche des Tadmait in ungefähr $800 \mathrm{~m}$ Meereshöhe entspringt und in nordöstlicher Richtung zur Oase Ouargla zieht? Dieser Oued führt in der Tat heute noch Wasser, und zwar alle ein oder zwei Jahre durchschnittlich einmal; sein Wasser legt dabei Laufstrecken von bis zu $400 \mathrm{~km}$ Länge zurück ${ }^{5}$. Nördlich von Ouargla ist das Tal kaum mehr erkennbar. Hat der Oued Mya zur saharischen Pluvialzeit vielleicht seine Wasserfluten über Touggourt hinaus in den Schott Melrhir gewälzt und damit eine Länge von $900 \mathrm{~km}$ erreicht? Oder ist das Gebiet des Oued Rhir als der alte, seit der Pluvialzeit verfallene und verkümmerte Unterlauf des Oued Igharghar anzusehen, der gleichfalls aus dem Süden kommt, und zwar aus dem Herzen der Sahara, dem Hoggar-Gebirge? Heute indessen neigt man, namentlich auf Grund geologischer Untersuchungen, eher der Ansicht zu, daß es sich im Oued Rhir um ein Einbruchsfeld handelt, das mit dem Igharghar in keinem ursächlichen Zusammenhang steht. Es wäre somit, entgegen seiner Bezeichnung, gar kein altes Flußtal. Die Araber pflegen, wie Hauptmann Bajolle ${ }^{5}$ bemerkt, den Namen Oued gelegentlich auch vollständig geschlossenen und jeglichen Wasserfließens baren Niederungen zu geben, falls sie durch ihre Form und Ausdehnung die Illusion erwecken, alte Flußlinien zu sein. Im Oued Rhir hat jedenfalls seit Menschengedenken kein Wasserfließen mehr stattgefunden.

Wie alle Teile der Sahara, so zeichnet sich auch die Region des Oued Rhir durch große Sommerhitze (maximale Schattentemperaturen von $50^{\circ}-55^{\circ}$ ) und große Trockenheit aus. Die Berechnungen aus den Meßergebnissen einer vierzigjährigen Beobachtungszeit (1913-53) haben ergeben, daß das jährliche Niederschlagsmittel bloß 60 Millimeter beträgt. Als Maximum sind während dieses Zeitraumes, und zwar im Jahre 1933, 119,6 Millimeter, also gerade das Doppelte, festgestellt worden. Demgegenüber sind aber Jahre mit weniger als 10 Millimetern nichts seltenes. Begreiflich darum, daß die Gärten künstlich bewässert werden müssen. Diesem $Z$ wecke dienen die artesischen Brunnen, die Grundwasser an die Oberfläche befördern. Sie kommen in so großer Zahl vor, daß die Landschaft und Bewässerungswirtschaft durch sie ihr besonderes Gepräge erhalten.

Dieses "Land der artesischen Brunnen" weist mehrere, in miopliozäne Sande und Kalke eingeschlossene Grundwasserschichten auf, die durch undurchlässige Gesteinsschichten, wie Mergel und Tone, voneinander getrennt werden. Eine erste Wasserschicht findet sich fast überall bloß wenige Meter unter der Erdoberfläche, im allgemeinen deren 3-10. Sie wird auf verschiedene Arten gespeist, so durch die Niederschläge, durch das Sickerwasser aus Gärten und Brunnen und durch die Wasseraufstöße, die aus tieferliegenden Wasserhorizonten längs Spalten erfolgen. Wasser wird ihr auch von den Behars geliefert, den kleinen Seen und Wasserlachen, die durch Einsturz alter aufgegebener Brunnen entstanden sind. Am wichtigsten ist mit 14 ha Fläche der $40 \mathrm{~m}$ tiefe Behar von Merdjadja, der $6 \mathrm{~km}$ südlich von Touggourt liegt. Das Wasser dieser obersten Schicht ist sehr 
salzhaltig, namentlich an Natrium, Magnesium und Gips, und darum als Trink- und Bewässerungswasser ungeeignet. Der Salzgehalt beträgt je Liter 3,5-7,8 g.

Dieser Wasserschicht verdanken die abflußlosen Schotts ihr Dasein, die in großer Zahl in der eintönigen Niederung des Oued Rhir vorkommen, und zwar immer an ihren tiefstgelegenen Stellen, denn nur hier vermag das Grundwasser zutage zu treten und viele Quadratkilometer Land $\mathrm{zu}$ überschwemmen. Die Schotts sind nach einem Ausdruck von $\mathrm{H}$. Schiffers ${ }^{6}$ nichts anderes als « der sichtbar gewordene Spiegel des Grundwassers ». Sie sind nicht tief, selten mehr als einen Meter, und in ihrer Ausdehnung starken Veränderungen unterworfen. Mit Eintritt der warmen Jahreszeit, von März an, wenn die Verdunstung stärker wird, beginnen sie zu schrumpfen und im Laufe des Sommers ver-

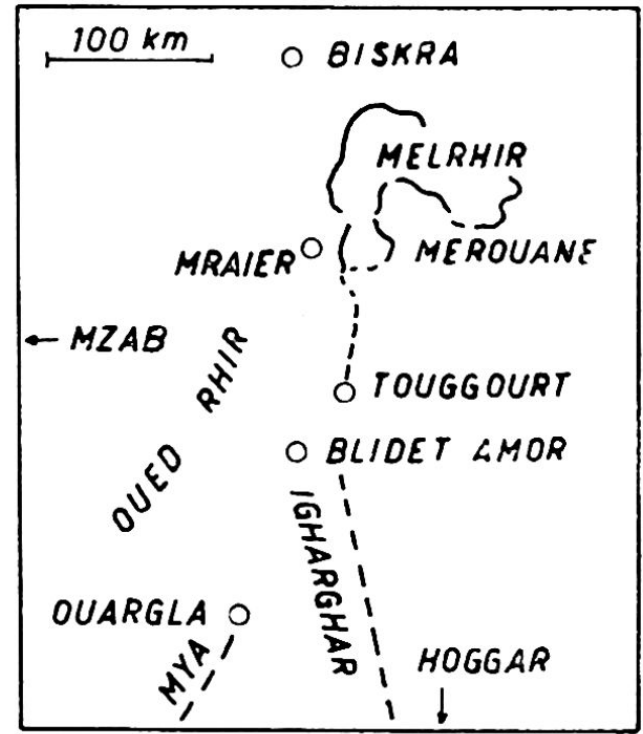

Orientierungsskizze des Oued Rhir schwinden sie ganz. An diesem Vorgang haben auch die Winde ihren Anteil. Berechnungen über die Größe der Verdunstung liegen bis heute keine vor. F. PAIX ${ }^{7}$ veranschlagt sie auf ungefähr $50 \mathrm{~cm}$ im Jahr. Auf Grund dieser Schätzung ergäbe sich für das ganze Gebiet des Oued Rhir, dessen Schotts zusammen eine Fläche von $2000 \mathrm{~km}^{2}$ bedecken, ein Wasserverlust von $30 \mathrm{~m}^{3}$ in jeder Sekunde. Im Sommer breiten sich an Stelle der Wasserflächen weißschimmernde Salzkrusten aus. Sie werden von den Eingeborenen an gewissen Stellen zur Salzgewinnung ausgenützt. Im Winterhalbjahr, von November an, bilden sich die Schotts wieder. Die oberste Wasserschicht ist also in ihrer Mächtigkeit starken jahreszeitlichen Schwankungen unterworfen.

Als Wasserlieferanten kommen nur die tiefer gelegenen Schichten in Frage. Es sind vor allem deren zwei, die eine davon zwischen $20-40 \mathrm{~m}$ Tiefe gelegen und die andere unter $50 \mathrm{~m}$; höchstens aber in $160 \mathrm{~m}$. Beide Schichten werden in zahlreichen Brunnen ausgenützt. Der Wasserertrag dieser Brunnen ist sehr verschieden, bei jenen der oberen Schicht minimal 50 Minutenliter und maximal 1000, und bei jenen der unteren Schicht 200 bezw. 2000. Doch im mittleren Abschnitt des Oued Rhir ist namentlich der obere Wasserhorizont (10-40 $\mathrm{m}$ Tiefe) ergiebig und ebenso im untern Abschnitt (20-50 m Tiefe). Die Zahlen für die Brunnen lauten 1000-7000 Minutenliter für den mittleren Abschnitt und 2000-15000 für den untern. Die Grundwasservorkommen, deren geologische Verhältnisse im einzelnen recht kompliziert sind, halten sich an Sande und feinere Kiese, ausnahmsweise auch an Seekalke, alles Gesteine aus dem Pliozän und Miozän. Was die Herkunft dieses Grundwassers anbetrifft, so geht die Vermutung dahin, daß es von einem unterirdischen Strome herrühre, den die beiden Oued Mya und Igharghar speisen. Ferner dürfte ihm Wasser aus dem westlich des Oued Rhir gelegenen Kreideplateau des Mzab zufließen, dessen Trockenflüsse auch gelegentlich einmal Wasser führen. Die Grundwasserströme würden somit von Süden nach Norden und von Westen nach Osten dahinziehen.

Die Grundwasservorräte werden von den Eingeborenen seit dem 14. Jahrhundert ausgenützt. Als die Franzosen das Oued Rhir im Jahre 1854 besetzten, waren nicht weniger als 450 artesische Brunnen im Betrieb. Diese wurden auf ganz einfache Weise erstellt. Die Eingeborenen trieben mit Hacken ungefähr $1 \mathrm{~m}$ breite und bis $60 \mathrm{~m}$ tiefe, viereckige Schächte bis zum Dach der Grundwasserschicht, einer mergelig-kalkigen Gesteinsschicht, von ihnen Mahzoul geheißen, vor und kleideten diese mit Palmholz aus, nachdem vorher noch ihre Wände mit Ton und Palmbast (Lif) 
gut abgedichtet worden waren. So sollte vom aufsteigenden kostbaren Wasser ja nichts durch Versickern verloren gehen. Hierauf stieg der Brunnenmeister (Mallem) in den Schacht hinab und durchschlug das Dach. Sobald Wasser floß, kehrte er nach oben zurück. Nun galt es, den Brunnen zu reinigen, denn das durch den Mahzoul geschlagene Loch wurde stets durch Sand und Kies, den das aufschießende Wasser mit sich führte, mehrere Meter hoch verstopft. Diese schwierige Arbeit hatten die Taucher, die sogenannten Rtassins, zu besorgen. Miteinander abwechselnd stiegen sie in den Schacht hinab und füllten dort rasch einen Korb mit Schutt, der dann an einem Seil emporgezogen wurde.

Der Beruf der Taucher ist heute im Aussterben begriffen, denn seit 1949 dürfen keine solchen «Araberbrunnen » mehr erstellt werden wegen ihrer enormen Wasserverluste durch Versickern. Selbst der Bau von runden und mit Zement ausgekleideten Brunnen, dem sich die Eingeborenen in jüngerer Zeit zuwandten, ist verboten worden. Nur vereinzelt kann man noch einen Taucher an der Arbeit sehen, so z. B. im Gebiet von Temacine. Er tritt jedesmal dann in Aktion, wenn es einen alten Brunnen zu reinigen gilt. Gegenwärtig sind noch deren rund 150 in Betrieb. Die Rtassins vermögen bis in Tiefen von $60 \mathrm{~m} \mathrm{zu}$ tauchen und im Wasser drei bis fünf Minuten auszuharren. Das gelingt ihnen im Tag fünf- bis achtmal.

Der Araberbrunnen wird seit Jahren durch den viel besser gebauten Europäerbrunnen abgelöst, bei welchem Röhren in den Boden vorgetrieben werden. Die Wasserverluste durch Versickern sind bedeutend geringer. Gegenwärtig gibt es deren bereits 810, die mit den alten Brunnen zusammen eine Wassermenge von 301000 Minutenlitern liefern. Dazu kommen noch 20000 Minutenliter von 201 Brunnen, die nicht artesisch sind und deren Wasser man deshalb heraufpumpen muß. Das geschieht in 175 Fällen mit Hilfe von senkrecht stehenden Schöpfrädern (Noria), die von im Kreise herum laufenden Eseln oder Maultieren in Bewegung gesetzt werden, und in den restlichen 26 Fällen mit Hilfe der weit besser arbeitenden Motorpumpe. Sie kann leicht 1000 Minutenliter fördern, die Noria indessen bloß 300-400.

Das Gebiet des Oued Rhir verfügt alles in allem über 321000 Minutenliter Bewässerungswasser. Das macht im Durchschnitt für jede Hektare bebauten Bodens deren 32, ein Betrag, der von den Sachverständigen als ungenügend angesehen wird; es sollten 40 sein. Dieser beträchtliche Fehlbetrag an Wasser besteht, wie R. CAPOTREY 4 berichtet, seit 1935. Seit dieser Zeit ging der Ertrag manch eines Brunnens zurück. Heute wird aber alles daran gesetzt, um durch geeignete Maßnahmen einen weiteren Rückgang zu verhindern. So werden die alten Araberbrunnen auszementiert oder mit Röhren versehen. Einige wenige Oasen haben genügend Wasser, so jene von Mraier, wo auf jede Hektare im Durchschnitt 45 Minutenliter entfallen; andere indessen katastrophal wenig, wie z. B. jene von Meggarine-Tamerna mit bloß 14.

Eine Zeitlang glaubte man, nach Belieben neue Brunnen erstellen und im Zusammenhang damit auch neue Gärten schaffen zu können. Leider mußte man aber bald die Erfahrung machen, daß jede neue Bohrung, vor allem wenn sie in tiefer gelegenem Niveau erfolgte, den Wasserdruck und den Wasserertrag der benachbarten Brunnen herabsetzte, und zwar meist sehr schnell und empfindlich. So sank z. B. im Jahre 1937 schon 18 Stunden nach Erstellung des Brunnens von Tidjma in Tebesbest der Wasserspiegel im $2 \mathrm{~km}$ entfernten Brunnen des Bahnhofs von Touggourt um $44 \mathrm{~m}$. Stark war auch der Einfluß der im Jahre 1950 vorgenommenen Bohrung von Ourhir N'Sigha auf die beiden nur $6 \mathrm{~km}$ entfernten Brunnen von Steeg. Beim einen Brunnen, dem Ain Salar, ging der Wasserertrag plötzlich von 15400 Minutenlitern auf 13500 , d. h. um $12 \%$ zurück, und beim andern, dem Ain Bertin von 5125 auf 4630, d. h. um 9,6\%. Diese Feststellungen waren für die Eingeborenen und Europäer, die immer wieder darnach trachteten, durch neue Wasserbohrungen in tiefer gelegenem Gelände ihre Gärten auszudehnen, sehr schmerzlich. Wohl gelang es ihnen, dort neue Gärten ins Leben zu rufen, doch vermochten sie nicht, dem Absterben der alten, 


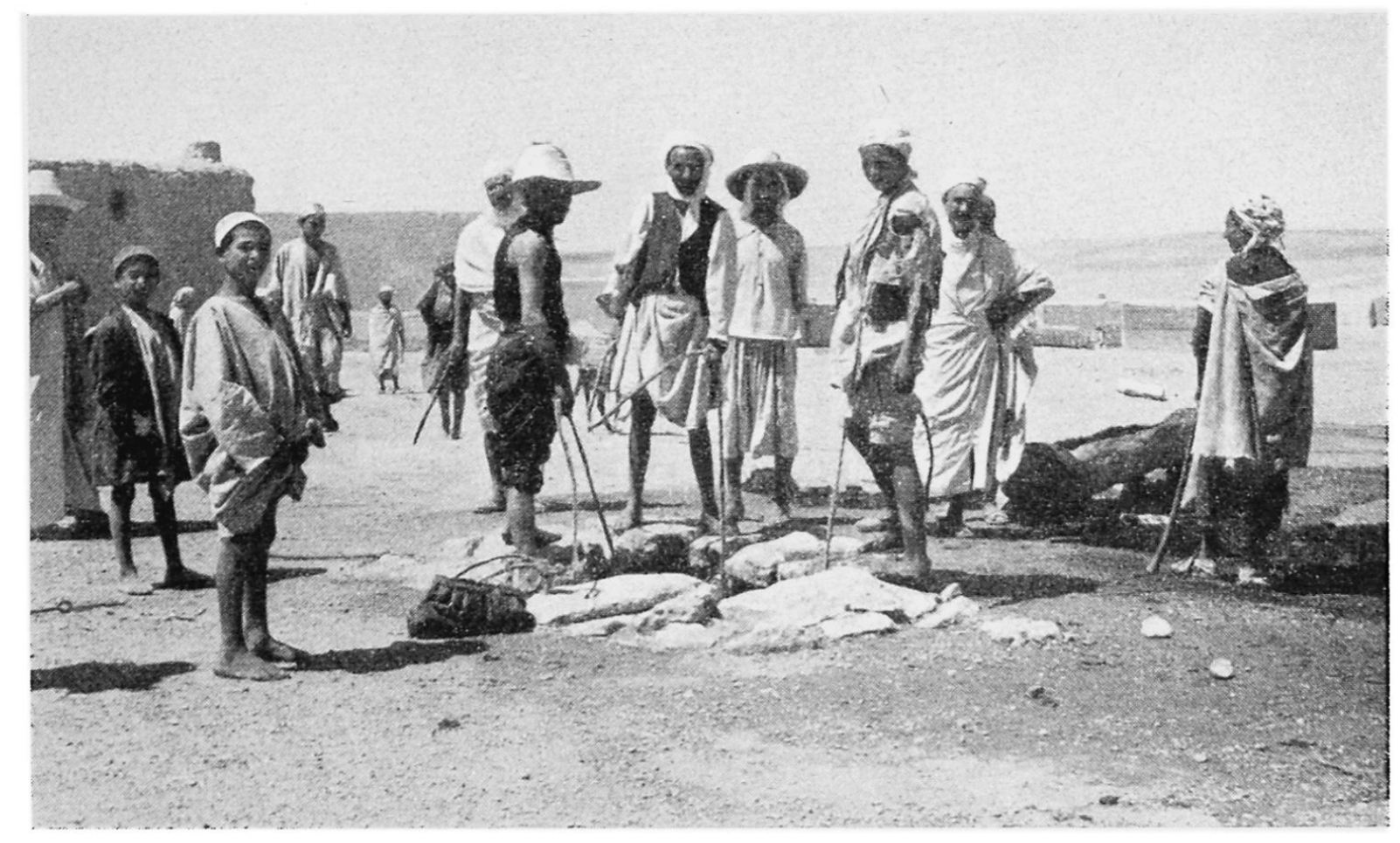

Brunnenreiniger an der Arbeit. Mit Seilen, Körben und Wassersäcken versehen, stehen sie um die Öffnung eines alten Brunnenschachtes. (Photo K. Suter)

höher gelegenen Gärten Einhalt zu gebieten. Es kam so in den Oasen des Oued Rhir in den letzten Jahrzehnten zu einem «Wandern »der Gärten von oben nach unten. Dennoch hat, im gesamten gesehen, die Wassermenge durch die neuen Bohrungen zugenommen und damit auch die Ausdehnung der Gärten. Heute zählt dieses Gebiet ungefähr 1300000 Palmen. Die Möglichkeiten zusätzlicher Wasserbeschaffung sind noch nicht erschöpft; nach Auskünften des Wasseramtes könnten allein dadurch, daß man die alten Brunnen ausbessern würde, noch weitere 100000 Minutenliter gewonnen werden.

Jeder Brunnen (Ain) hat seinen eigenen Namen, der oft an die Person, die ihn erstellen ließ, erinnert, wie Ain Sidi Sliman oder Ain Lougta, oder sonst an ein Merkmal, wie etwa die Bodenfarbe, so z. B. Ain hamra (roter Brunnen), oder Ain safa (gelber Brunnen). Die Brunnen haben meist viele Besitzer, in Meggarine z. B. einer 170. Ein Einzelner könnte sich den Bau eines ganzen Brunnens der großen Kosten wegen gar nicht leisten.

Das Wasser der Brunnen wird nach der Zeit verteilt. Jeder Teilhaber bekommt es in regelmäßig wiederkehrenden Intervallen eine bestimmte Zeit lang, entsprechend seinen Wasserrechten. Dem Verteilungsplan liegt eine Zeitspanne von 28 Tagen, bezw. von 28 Einheiten (Nouba) zugrunde. Wer eine ganze Nouba besitzt, was viel ist, bekäme das Wasser während einer solchen Periode einmal den ganzen Tag lang; doch wird er sich aus naheliegenden Gründen hüten, alles Wasser auf einmal zu beanspruchen; viel klüger ist es, kleinere Wassermengen zu beziehen, dafür aber umso häufiger, z. B. jede Woche, und zwar immer am gleichen Wochentàg, einen Viertel (6 Stunden), dabei das eine Mal tags und das andere Mal nachts (1. Woche z. B. von 6-12 Uhr, 2. Woche von 18-24 Uhr; 3. Woche von 12-18 Uhr und 4. Woche von 24-6 Uhr). Die Nouba wird in $1 / 2$ (Neß), 1/4 (Reboua), 1/8 (Temen), 1/16 (Karouba) und 1/32 (Tounsia) unterteilt. Aus starken Quellen, im allgemeinen aus solchen von mehr als 1000 Minutenlitern, macht man, und zwar unter Wahrung der Zeitspanne von 28 Tagen, 56 Einheiten, mit andern Worten, es beziehen aus ihr gleichzeitig immer zwei Bauern Wasser, nämlich jeder eine Hälfte. Ein solcher Brun- 


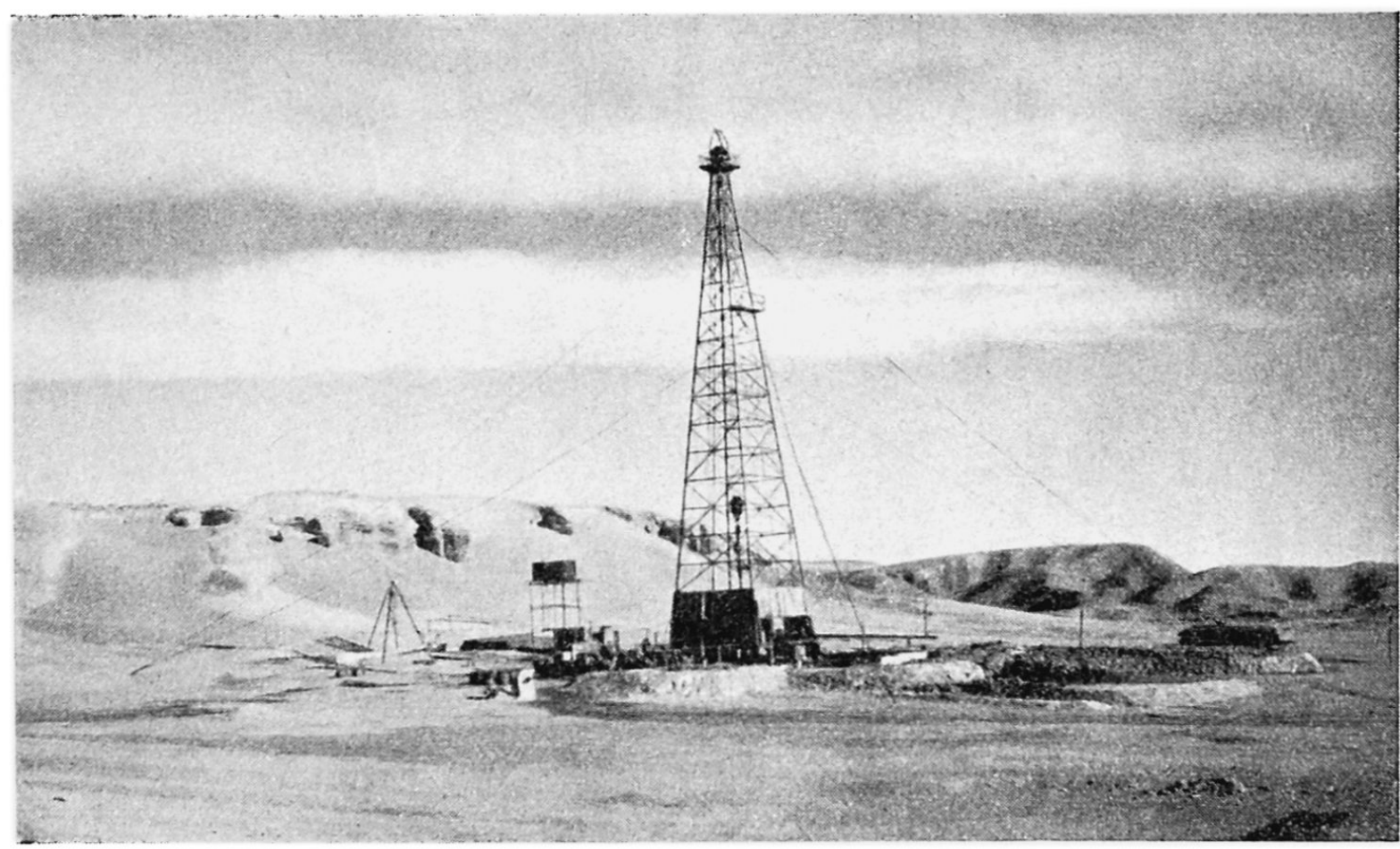

Der Service de la Colonisation et de l'Hydraulique in Algier führt seit 1927 in der algerischen Sahara Tiefenbohrungen durch, bei welchen es darum geht, das Wasser des Albien (mittlere Kreide) zu erreichen und an die Erdoberfläche zu befördern. Nachdem zwei in der Zeit zwischen 1927 und 1936 vorgenommene Versuchsbohrungen bei Touggourt und Ouargla, die bis auf etwas über $900 \mathrm{~m}$, indessen noch nicht bis ins Albien vordrangen, ergebnislos verlaufen waren, folgten einige weitere bis auf $1200 \mathrm{~m}$ Tiefe vorstoßende Bohrungen im benachbarten Mzab. Von besonderem Erfolg waren die beiden Bohrungen von Zelfana $(1946$ - 1948) und Guerrara (1948 - 1950), die dank starken Steigungsdruckes artesisches Wasser liefern. Die Bohrung von Guerrara, die mit Hilfe des im Bild sichtbaren $35 \mathrm{~m}$ hohen Bohrturms bis auf $1200 \mathrm{~m}$ hinunterging, begann in den Gesteinsschichten des Miozäns und führte hierauf durch die Schichten der oberen Kreide (Senon, Turon, Cenoman) in die Sande des Albien hinein. Der Wasserertrag beträgt mehr als 14000 Minutenliter. Man hofft, durch weitere Tiefenbohrungen im Gebiete des Oued Rhir, des Mzab und von Ouargla die Wasserschicht des Albien zur Wiederbelebung absterbender und zur Gründung neuer Oasen nutzbar machen zu können. (Photo K. Suter)

nen weist zwei Wasserableitungsgräben (Seguia) auf. Der Wasserbesitz ist nicht an den Boden gebunden; das Wasser kann deshalb unabhängig von diesem verkauft oder vermietet werden. Im Winterhalbjahr hat die Bewässerung jede Woche einoder zweimal zu erfolgen, im Sommer (April-September) indessen so oft, als es das zur Verfügung stehende Wasser überhaupt erlaubt. Im Herbst, nachdem die Sommergemüse eingebracht sind, kann auf die Berieselung eine Zeitlang verzichtet werden. Dann fließt das Bewässerungswasser leider ungenützt ab, weil man noch über keine Einrichtungen verfügt, um es für den Sommer aufzuspeichern.

Im Gebiete des Oued Rhir ist es nötig, die Gärten zu drainieren, d. h. das überschüssige und verbrauchte, mit Bodensalzen gesättigte Bewässerungswasser (Nezz), das die Humusschicht passiert hat, abzuleiten. Wo das nicht geschieht, bilden sich unliebsame, seuchengefährliche Wasserlachen, die ihres Salzgehaltes wegen mit der Zeit auch ein Absterben der Gärten zur Folge hätten.

Der Wasserableitung dienen besondere, $1-2 \mathrm{~m}$ tiefe Gräben (Khandeggs), die entweder durch die Gärten hindurchziehen oder deren Rändern entlang verlaufen. Über sie führen auf kurzen Holzbrücken die Wege und in ausgehöhlten Baumstämmen die Bewässerungsgräben. Das Wasser stagniert häufig in ihnen, so daß sich hier Brutstätten von allerhand Krankheiten, z. B. der Malaria, bilden können. Die Verhältnisse wurden, wie D. Moulias 2 berichtet, in einigen inmitten der Gärten gelege- 
nen Siedlungen vor Jahren so unhaltbar, daß diese aufgegeben werden mußten. Die Eingeborenen erstellten neue Wohnstätten außerhalb der gefährlichen Bewässerungszone. Die Verwaltung nahm den Kampf gegen das stagnierende Wasser auf, indem sie in den Jahren 1925-32 durch den Bau eines $90 \mathrm{~km}$ langen Hauptkanals, der das Wasser aller Khandeggs zu sammeln hat, für eine bessere Wasserableitung sorgte. Er beginnt bei der Oase Bou Yerrou im Südosten des Flugplatzes von Touggourt und endigt im Bett des Oued Krerouf. Dieses Bett wurde ausgebessert, so daß es ihm möglich ist, das Sammelwasser dem Schott Merouane zuzuführen. Während des Winterhalbjahres fließen diesem jede Sekunde durchschnittlich $5-6 \mathrm{~m}^{3} \mathrm{zu}$, im Sommerhalbjahr indessen fast nichts. Auch einzelne in der Nähe der Oasen gelegene Schotts wurden an den Hauptsammelkanal angeschlossen, so daß er auch einen Teil ihres Wassers ableiten kann. So wird begreiflich, daß sich das Kanalwasser durch einen großen Salzgehalt, nämlich $12-20 \mathrm{~g}$ je Liter, auszeichnet.

Die Privatgärten sind von ungefähr 1,2 $\mathrm{m}$ hohen Mauern, die aus Klumpen salzhaltiger Gartenerde bestehen, umgeben. Am wichtigsten ist die Dattelproduktion; das Oued Rhir erzeugt jährlich im Durchschnitt 150000 q. Am meisten geschätzt ist die Deglet nour, die bei sorgfältiger Pflege, namentlich ausreichender Düngung und Bewässerung, bestens gedeiht. Die maximalen Erträge je Baum beziffern sich auf 200 Kilo. Einen besonders guten Eindruck machen die neuen Gärten mit ihren ganz regelmäßig angeordneten Baumreihen, so jene von El Arfiane und Mraier. Neben der Palme spielt der Anbau anderer Gewächse, wie Getreide (Korn, Gerste), Gemüse (Rüben, Tomaten, Pfeffer, Bohnen; in den Europäergärten auch Spargeln und Artischoken) und Futterpflanzen (Klee, Luzerne) nur eine untergeordnete Rolle.

\title{
LITERATUR
}

1 Rolland, G.: Hydrologie du Sahara algérien. Paris 1895. - 2 Moulias, D. : L'organisation hydraulique des oasis sahariennes. Alger 1927. - 3 SAvORNIN, J.: Le plus grand appareil hydraulique du Sahara. Travaux de l'Institut de Recherches Sahariennes. Alger 1947. - 4 Capot-Rey, R.: Le Sahara Français. Presses Universitaires de France. Paris 1953. - 5 Dubief, J.: Essai sur l'hydrologie superficielle au Sahara. Gouvernement Général de l'Algérie, Alger 1953. Darin Zitat von Hauptmann Bajolle, S. 318. - 6 Schiffers, H.: Die Sahara. Franckh'sche Verlagshandlung. Stuttgart 1950. - 7 Der Verfasser verdankt einige Auskünfte und Zahlen dem Amt für Wasserwirtschaft in Touggourt.

\section{L'IRRIGATION ARTIFICIELLE DANS L'OUED RHIR}

Les connaissances sur l'origine de la contrée du Oued Rhir sont aujourd'hui encore hypothétiques. L'on suppose qu'elle s'est formée par suite d'un effondrement ou qu'elle est la trace d'une faille. L'irrigation des nombreuses palmeraies avec leurs 1300000 arbres s'effectue à l'aide de plusieurs centaines de puits artésiens. Leur eau provient de couches situées parfois à un niveau de $160 \mathrm{~m}$ de profondeur. Depuis nombre d'années, les anciens puits arabes, très primitifs, ont été remplacés par le « puit européen " mieux construit et plus solide. Cependant, le nombre ne peut en être augmenté volontairement, car chaque nouveau forage diminue l'apport en eau des anciens puits voisins, creusés dans un niveau légèrement plus élevé. Après avoir traversé les jardins, l'eau d'irrigation est salée et inemployable. Son surplus se déverse par des rigoles dans un canal qui mène au Chott Mérouane.

\section{DIE TAGUNG ZUM STUDIUM DER QUARTÄR-CHRONOLOGIE IN AARAU 13./14. MÄRZ 1954}

\author{
Rudolf Merian und Erich Schwabe
}

Die heutige Landoberfläche verdankt zu einem großen Teil den glazialen und fluviatilen Gestaltungskräften des Quartärs ihr Antlitz, weshalb für die Geomorphologie das Studium des Quartärs, insbesondere dessen Chronologie, ein Zentralproblem darstellt. Aber auch die Geologen, Botani- 